EPJ Web of Conferences 49, 18028 (2013)

DOI: $10.1051 /$ epjconf/20134918028

(C) Owned by the authors, published by EDP Sciences, 2013

\title{
Performance of the ATLAS Tile Calorimeter
}

\author{
Shima Shimizu ${ }^{1, a}$ on behalf of the ATLAS Collaboration \\ ${ }^{1}$ Nagoya University, Furo-cho, Chikusa-ku, Nagoya, 464-8601, Japan
}

\begin{abstract}
The Tile Calorimeter is the central section of the ATLAS hadronic calorimeter at the Large Hadron Collider. It is a key detector for the measurement of hadrons, jets, tau leptons and missing transverse energy. Because of its very good signal to noise ratio it is also useful for the identification and reconstruction of muons. The calibration and performance of the calorimeter have been established through test beam measurements, cosmic ray muons and the large sample of $p p$ collisions. Results on the calorimeter performance are presented, including the absolute energy scale, time resolution, and associated stabilities.
\end{abstract}

\section{The ATLAS Tile Calorimeter}

The ATLAS Tile Calorimeter (TileCal) [1] is the central hadronic calorimeter of the ATLAS detector [2] at the Large Hadron Collider (LHC). It is a sampling calorimeter using plastic scintillator tiles as active material and steel plates as absorber. The calorimeter has a total length of 12 $\mathrm{m}$ with a diameter of $8.5 \mathrm{~m}$ and its total thickness is 7.4 $\lambda_{\text {int }}$ at $\eta=0$. It consists of three cylinders, one long barrel (LB) splitted into two readout partitions, LBA and LBC, and two extended barrels (EBs), EBA and EBC, covering $|\eta|<1.7$.

Each barrel is divided into 64 modules in azimuth, $\phi$, giving a granularity of $\Delta \phi \sim 0.1$. A schematic view of a module is shown in Figure 1 (a). Each module is further radially segmented in three layers of readout cells with a granularity of $\Delta \eta \sim 0.1$ for the first two layers and $\Delta \eta \sim$ 0.2 for the third one. The cell segmentation is shown in Figure 1 (b) for a half of LB module and an EB module (LBA and EBA). In total, TileCal has 5182 cells. Each cell is read-out by two photomultiplier tubes (PMTs) from either side of it, except for special cells, via wavelength shifting fibres.

TileCal is equipped with several systems to monitor and to calibrate each step of the readout: front-end readout circuit, PMTs and optics. The Charge Injection System [3] injects a well defined charge to the readout electronics to derive $\mathrm{ADC}$ to $\mathrm{pC}$ conversion factors, $C_{\mathrm{ADC} \rightarrow \mathrm{pC}}$, with $0.7 \%$ precision. The laser system [4] sends light pulses to all the PMTs to monitor the PMT gain of each channels, giving a calibration constant $C_{\text {laser, }}$, with $1 \sim 2 \%$ precision. It is also possible to derive timing constants with the laser system. The optics are monitored by the $C_{S}$ system [5], where a radioactive cesium source traverses through all the tiles in order to equalize the response between individual channels and to monitor stability of optics elements. It gives a calibration constant, $C_{\mathrm{C}_{\mathrm{S}}}$, with

\footnotetext{
a e-mail: shima.shimizu@ cern.ch
}

$0.3 \%$ precision. The energy deposited in TileCal is reconstructed by applying these calibration constants as

$$
E[\mathrm{GeV}]=A[\mathrm{ADC}] \cdot C_{\mathrm{ADC} \rightarrow \mathrm{pC}} \cdot C_{\text {laser }} \cdot C_{\mathrm{CS}_{\mathrm{S}}} \cdot C_{\mathrm{pC} \rightarrow \mathrm{GeV}},
$$

where $E$ is the reconstructed energy in $\mathrm{GeV}$ and $A$ is the signal amplitude in ADC counts. The last constant $C_{\mathrm{pC} \rightarrow \mathrm{GeV}}$ is a factor to convert charge to electromagnetic scale energy and was determined from the test beam measurements in 2001-2003 using electron beams [6].

\section{Operation of the Tile Calorimeter in the year 2012}

In case of a serious failure of on-detector electronics, TileCal cells might require to be turned off for smooth data taking. During the ATLAS data taking in the year 2012, fraction of non-usable cells was less than $\sim 2.5 \%$. These non-usable cells are mostly due to 4 half LB modules that were not operational. The energy deposited in these cells is recovered by interpolation from their neighbouring cells during the energy reconstruction, thanks to the fine granularity of TileCal and the fact that these modules are not neighbouring. Figure 2 shows the time evolution of the fraction of unusable cells starting from the beginning of the year 2011. The figure shows that the masked cells, which represented $5 \%$ of the total at the end of the 2011 operation, were taken care of during the winter maintenance period 2011/2012 and there were less failures during the 2012 operation than in 2011.

Another improvement during the maintenance period is given by replacement of the Low Voltage Power Supplies (LVPS). These are installed on the front-end electronics and located in the detector hall, in a high radiation environment. Frequent power trips were observed during the 2011 operation. A revised design of the LVPS [7] was installed in 40 modules. In addition to solving the problem of the frequent trips, the new LVPS also reduces the 


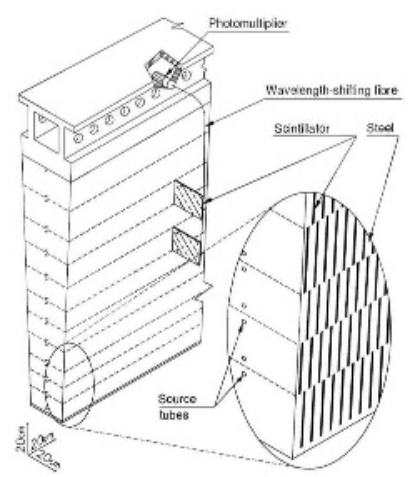

(a) Module structure

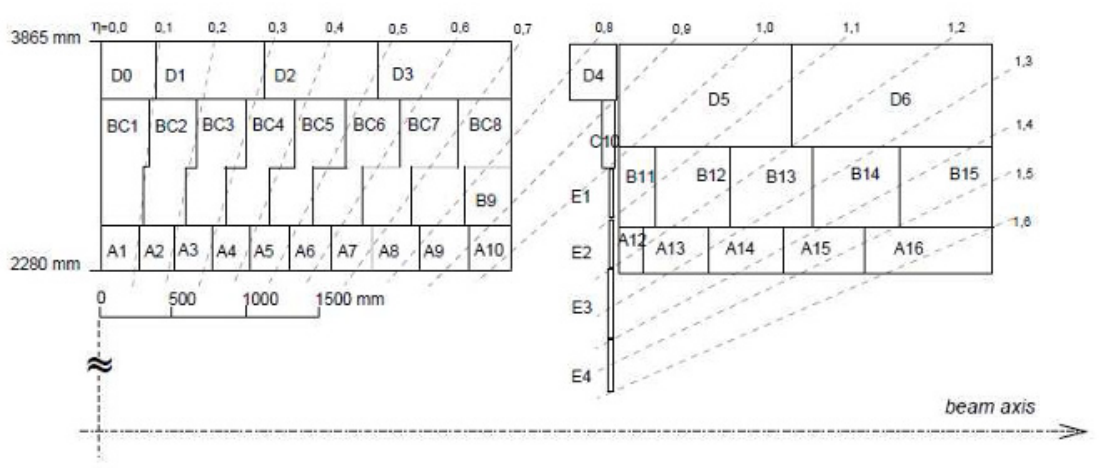

(b) Cell segmentation

Figure 1. (a) A schematic view of a module, showing its structure and its signal collection system. (b) A schematic view of a half of the calorimeter along the beam-axis, showing a half LB module and an EB module, with the cell segmentation. Cells are aligned in three layers, $\mathrm{A}, \mathrm{BC}$ and $\mathrm{D}$ for $\mathrm{LB}$ modules and $\mathrm{A}, \mathrm{B}$ and $\mathrm{D}$ for $\mathrm{EB}$ modules.

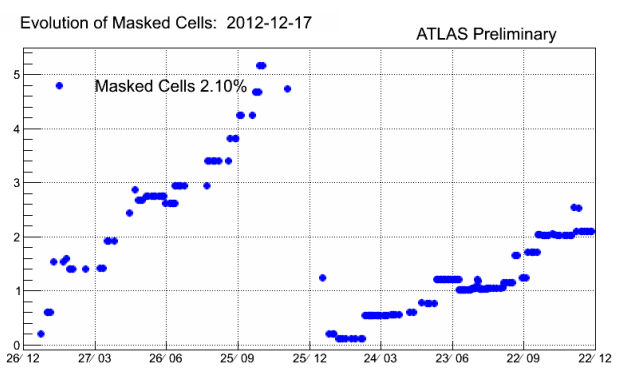

Figure 2. Evolution of the amount of "masked", i.e. unusable, cells shown from the beginning of the 2011 operation (26/12/2010) to the end of the 2012 operation (22/12/2012) [8].

non-gaussian component of the noise distribution, thus resulting in a reduction of the noise values.

\section{Performance}

\subsection{Performance with non-collision data}

Energy deposits from cosmic rays allows the study of cell responses to muons, as the interaction of muons with steel is generally well understood. The response is estimated by a truncated mean of muon energy loss per unit path length, $d E / d X$, in which $1 \%$ of a tail with high $d E / d X$ is removed. The energy loss is measured by TileCal and the path length is defined by muon tracks reconstructed with the ATLAS inner tracking detector and extrapolated to the calorimeter. Uniformity of cells is studied and found to be within $\pm 3 \%$ in a layer. It is shown in Figure 3 for a layer in LB modules, together with a comparison to the Monte Carlo simulation, which describes the data well. The maximum difference between layers is found to be $4 \%$. The studies were done using cosmic muon data collected in 2008, 2009 and 2010 separately and a stable response was obtained. The uncertainties on the studies are less than $1 \%$ for the layers in LB modules and less than $3 \%$ for the layers in EB modules.

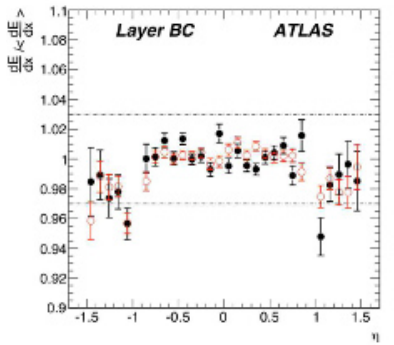

(a) $\eta$ dependence

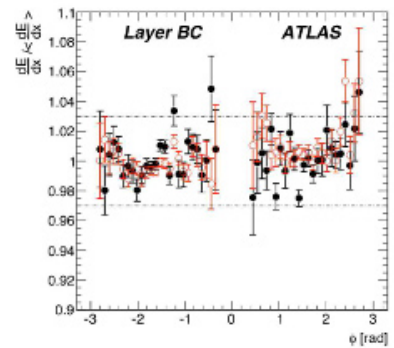

(b) $\phi$ dependence
Figure 3. Cell energy uniformity using cosmic muon rays in BC layer in LB modules [1]. Data (closed circles) are well reproduced by Monte Carlo predictions (open circles). The horizontal lines limit the $\pm 3 \%$ bands.

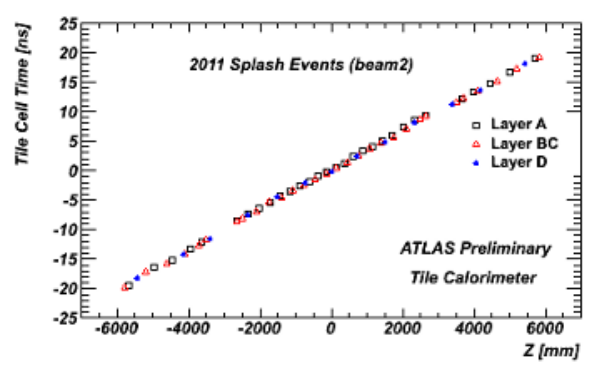

Figure 4. Timing of signals easured with splash events shown for each cell as a function of the cell $z$ coordinate along the beam axis [8].

Special single-beam runs cause splash events, in which many particles travel through TileCal almost parallel to the beam axis. Synchronisation of cell timing can be checked with such events as seen in Figure 4, which shows the linear correlation between signal timing and $z$ coordination, which is along the beam axis, of cells. After time-of-flight correction, very good timing equalisation, within $1 \mathrm{~ns}$, was obtained. 


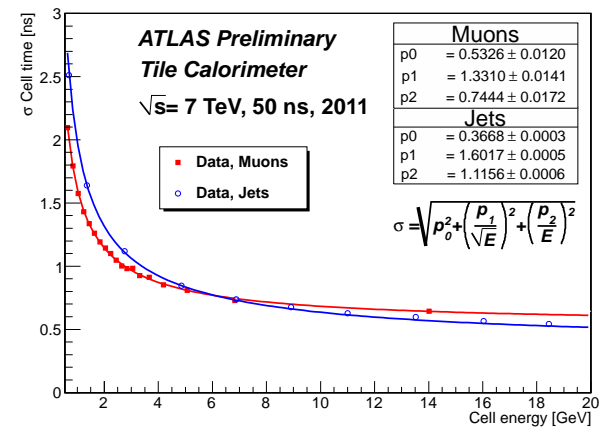

Figure 5. Cell time resolution as a function of cell energy in the 2011 collision data at $\sqrt{s}=7 \mathrm{TeV}$ and $50 \mathrm{~ns}$ bunch spacing, shown for muons and jets [9]. The lines are parametrised resolution as a formula in the plot.

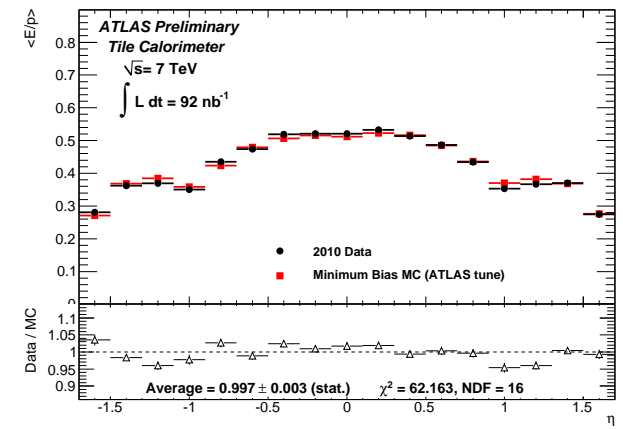

Figure 6. Mean value of the $E / p$ as a function of $\eta$, integrated over all $p$ ranges for 2010 collision data and Monte Carlo simulation [9]. Bottom plot shows ratio of data to Monte Carlo of the mean value of the $E / p$. The value shown is a fitted value with a constant function. The error shown is only statistical.

\subsection{Performance in beam collisions}

Excellent timing resolution is required to distinguish signals from different events close in time or to tag outof-time energy deposits coming from non-collision background or previous bunch crossings. The timing resolution was measured as a function of energy deposit for muons and jets using $\sqrt{s}=7 \mathrm{TeV} p p$ collision data. The result is shown in Figure 5. The resolution gets better for higher cell energy and is found to be less than $1 \mathrm{~ns}$ for $E>3 \mathrm{GeV}$ for both muons and jets. It is well described by the parametrisation reported in the figure.

The calorimeter energy response is also investigated using isolated charged hadrons. Isolated tracks having energy deposits compatible with minimum ionising particles in the electromagnetic calorimeter, which is in front of TileCal, are selected. Their energy, $E$, deposited in TileCal is compared with their momentum, $p$, measured in the tracking detectors, giving $E / p$. Figure 6 shows the mean values of $E / p$ as a function of $\eta$ for the $\sqrt{s}=7 \mathrm{TeV}$ $p p$ collision data and Monte Carlo simulation. The data is reasonably described by Monte Carlo within $\pm 5 \%$.

\section{Summary}

During data taking at the LHC, the ATLAS Tile Calorimeter has demonstrated its good performance. The calorimeter is equipped with several calibration and monitoring systems that ensure the time stability of the calorimeter response and its uniformity with good precision. Its operation in the year 2012 was successful with improved detector conditions. Energy and timing reconstruction were checked using several non-collision data and beamcollision data. The time synchronisation between cells is found to be well within $1 \%$ from splash events. The uniformity of energy response is found to be within $4 \%$ and is stable for three years based on cosmic muon data. Very good timing resolution is seen in $p p$ collisions and energy response to isolated single particles in collisions are well described by Monte Carlo simulation.

\section{References}

[1] ATLAS Collaboration, "Readiness of the ATLAS Tile Calorimeter for LHC collisions," Eur. Phys. J. C 70 (2010) 1193

[2] ATLAS Collaboration, "The ATLAS Experiment at the CERN Large Hadron Collider,' JINST 3 (2008) S08003.

[3] K. Anderson et al., "Design of the front-end analog electronics for the ATLAS tile calorimeter," Nucl. Instrum. Meth. A 551 (2005) 469.

[4] S. Viret [LPC ATLAS Collaboration], "LASER monitoring system for the ATLAS tile calorimeter," Nucl. Instrum. Meth. A 617 (2010) 120.

[5] E. Starchenko et al., "Cesium monitoring system for ATLAS Tile Hadron Calorimeter," Nucl. Instrum. Meth. A 494 (2002) 381.

[6] P. Adragna et al., "Testbeam studies of production modules of the ATLAS tile calorimeter," Nucl. Instrum. Meth. A 606 (2009) 362.

[7] G. Drake et al., "An Upgraded Front-End Switching Power Supply Design for the ATLAS TileCAL Detector of the LHC," ATL-TILECAL-PROC-2011-015.

[8] "Approved Tile Calorimeter Plots", https://twiki. cern.ch/twiki/bin/view/AtlasPublic/ApprovedPlotsTile

[9] "Public Tile Calorimeter Plots for Collision Data", https://twiki.cern.ch/twiki/bin/view/AtlasPublic/ TileCaloPublicResults 